\title{
JOB RECOMMENDATION USING FACEBOOK PERSONALITY SCORES
}

\author{
Thiam Li Ting ${ }^{1}$, Kasturi Dewi Varathan ${ }^{2}$ \\ ${ }^{1,2}$ Department of Information Systems \\ Faculty of Computer Science and Information Technology \\ University of Malaya, \\ 50603 Kuala Lumpur \\ Malaysia \\ Email:thiam216@siswa.um.edu.my ${ }^{1}$, kasturi@um.edu.my² \\ DOI: https://doi.org/10.22452/mjcs.vol31no4.5
}

\begin{abstract}
Facebook is one of the most popular social media sites that has become part of our lives. User-generated Facebook data are useful and can be used to gauge personality. However, previous studies did not use Facebook data for personality assessment and mapping for professional purposes. The current study mainly aims to identify personality features using user-generated content in Facebook. A computational score is created and a model is developed by utilizing these scores in job recommendations that match the personality of the user. The personality score of Facebook is benchmarked against the Big Five Inventory (BFI) test score to determine accuracy. The scores of Facebook personality scores and BFI test reached 93.1\%. The findings of this study benefits job candidates, especially fresh graduates by assisting them in identifying a career that suits their personality. This study also helps create awareness among individuals by identifying the personality strengths and weaknesses through the use of Facebook information. This study can help employers find candidates who fit the needs of the company by gauging their personality through Facebook data.
\end{abstract}

Keywords: Facebook, personality, big five model, software engineering jobs

\subsection{INTRODUCTION}

Social media users have increased dramatically in recent years. Social media plays an important role in our lives and has become a vital tool for interpersonal communication and interaction. Popular social media sites include Facebook, Twitter, YouTube, Linkedin, Instagram, and Google Plus. As of December 31, 2015, Facebook recorded 1.59 billion monthly active users worldwide and 1.44 billion mobile monthly active users. Approximately $83.6 \%$ of daily Facebook users live outside the USA and Canada [20].

Facebook has become indispensable since its inception in 2004. Every day, people spend a significant amount of time on Facebook sharing and communicating with one another through online activities, such as liking, commenting, or sharing status updates. A survey by Statisticsbrain shows that each user spends an average time of 18 minutes per Facebook visit [21]. At present, 13.3 million or approximately $45.5 \%$ of Malaysians use Facebook. The majority of these users fall under the age group of 18-24 (34.5\%), followed by those 25-34 years (29.5\%) and those in the 13-17 age group (16.3\%) [11]. Activities and information on Facebook reflect the personality of the user [7]. Previous research suggested that the Facebook usage can predict the personality of users [5][6][7]. However, existing studies did not apply such information in computing personality scores. These studies primarily focused on discovering the positive or negative relationship between personality and features. In the present study, user-generated Facebook data were collected and utilized to gauge the personality of users.

Personality is described as the feelings a person feels, his or her thoughts, and behavior patterns. Each person has a unique personality that differentiates him or her from other people [35]. Personality reveals the different reactions, responses, and feelings of people in different situations and environments. In other words, people with different personalities will behave differently in similar situations. Personality tests are the usual means of assessing an individual's personality. Examinees answer questions or items in a personality test by evaluating themselves based on each of the items that reflect their behavior. The Big Five Inventory (BFI) Test is a commonly used personality test that contains 44 questions. These questions assess a personality through the Big Five Model. However, the BFI 
Test is a tedious means to determine a user's Big Five personality traits. The major disadvantage of the BFI Test is the tendency of users to be extra cautious in answering the survey questions thereby resulting in misleading results [27].

Personality is one of the main criteria used to select a career path for job seekers, especially fresh graduates [44]. Research that uses personality for job matching chiefly emphasizes offline situations through personality tests. This approach neglects the mapping of social media data. Existing systems at present cannot automatically retrieve usergenerated Facebook data to match appropriate jobs with personality. Personality is a crucial factor that is often overlooked during employment selection in addition to educational background and skills [46].

According to the National Education Statistics of Higher Education in Malaysia, 25\% of graduates in 2013 reported unemployment after graduation. Figures indicate that 4,328 out of 18,000 students $(24 \%)$ in the field of Information and Communication Technology were unemployed. Students continued to encounter employment problems despite their educational background [43]. The survey also shows that unemployed graduates are mostly the Bachelor's degree holders. A vital determinant of unemployment is the mismatch between the job demand and the personality of job seekers [32]. A recent Jobstreet.com report suggests that the mismatch between personality and career is among the top reasons for unemployment among graduates [44]. Job seekers, especially new graduates, may end up jobless or assume jobs that are incompatible with their strengths and capabilities. Problems arise because of the failure of graduates to realize their own capabilities and their failure to note the degree of suitability of their job with their personality. Personality should be considered when finding the right employment.

In this study, personality scores are mapped against jobs in the field of Software Engineering. This study is limited to Software Engineering jobs; this line of work was identified as one of the top employment by the Institute of Labour Market and Information and Analysis (ILMIA), a division set up in May 2012 by the Ministry of Human Resource Malaysia [23].

The foremost contribution of this study is to predict the personality of Facebook users through an analysis of their Facebook activities. A system was developed to evaluate the personality through Facebook data; the result is used to provide recommendations that suit the user's personality. Existing studies on personality prediction in $[1][2][3][4][5][6][7]$ used social media data and omitted the computation of personality scores. By contrast, the present study manages performs such calculation to assess user's personality through user-generated Facebook data. A proposed scoring mechanism computes the Facebook personality scores (FPSs) of an individual based on Facebook features. The outcome of this study aims to assist users in determining the most suitable jobs in software engineering by gauging their personality through an analysis of Facebook data. The accuracy obtained for FPSs for 30 respondents was $92.5 \%$ and $93.1 \%$ for 50 respondents.

The following sections discuss related works, methodology, results, and conclusions.

\subsection{RELATED WORKS}

Social media has become a vital means for social interactions for the past 10 years. Facebook is one of the most popular social media with more than 1 billion members. Facebook allows members to connect with one another by updating their status, providing comments, uploading photos, and joining groups and other activities. Previous research indicated that Facebook features can predict the personality of a user [5][6][7]. An uploaded photo, a shared wall post, or group membership in Facebook can reflect the personality of an individual [7]. Unfortunately, no existing mechanism can measure the strength of each personality trait. Past studies only focused on identifying the relationship between Facebook features and personality traits [1][2][3][4][5][6][7].

Past research suggested that each personality trait in the Big Five Model shows a positive or negative relationship with Facebook features [1]. Facebook features, such as friends, groups, photos, likes, and others, reflect the personality of a user. seven features were chosen in the present study, which includes friends, albums, wall posts, likes, comments, photos, and groups; these features were selected on account of their high usage [18][19][20][21][22]. Conclusions from [7] state that openness to experience is positively correlated with the number of status updates, photos, groups, and likes [7]. Past studies ([1],[2],[3],[4],[5], and [6]) show that highly extraverted people have several friends on Facebook. According to [7], people who are high in extraversion are likely to connect with people and have increased interactions with others using Facebook groups. From [10], we can find that individuals with high conscientiousness upload fewer photos than those with low conscientiousness. According to [6] and [8], people with high ratings of agreeableness have a positive correlation with comments 
posted. Facebook users with high neuroticism tend to use words to express negative emotion and anger in their status updates [9].

Some of the personality models commonly used in predicting personality include the Big Five Model, Holland's model, and the MBTI model. Holland's model, which is also known as the RIASEC model, consists of the following personality traits: Realistic, Investigate, Artistic, Social, Enterprising, and Conventional. Holland's model is frequently applied to measure job satisfaction in relation with factors, such as interests and environment [28]. The Myer-Briggs Type Indicator (MBTI) is a popular instrument used to measure and identify individual personality types. The four-dimensional pairs in the MBTI model include extraversion (E) and introversion (I), sensing (S) and intuition $(\mathrm{N})$, thinking $(\mathrm{T})$ and feeling $(\mathrm{F})$, and judging $(\mathrm{J})$ and perceiving $(\mathrm{P})$ [25]. Holland's Model and the MBTI model overlook Neuroticism in predicting personality. Thus, the Big Five Model was chosen instead of Holland's model or the MBTI model. The Big Five Model is generally accepted and applied by numerous established researchers [5]. The five personality traits in the Big Five Model include openness to experience (O), conscientiousness $(\mathrm{C})$, extraversion $(\mathrm{E})$, agreeableness $(\mathrm{A})$, and neuroticism $(\mathrm{N})$. People with openness are creative, curious, and possess unusual ideas [7]. Those with high levels of conscientiousness are usually self-controlled, hardworking, well-organized, and focused on their goals [7]. People with high ratings of extraversion tend to be active, assertive, and energetic [7]. People with high agreeableness people are kind, helpful, sympathetic, and cooperative [7][13]. Conversely, those with high neuroticism usually feel anxious, insecure, and are easily depressed $[5][7]$.

Past research used personality traits in different recommender systems. A recommender system helps a user make decisions in a complicated situation. Personality was applied in relation to purchase preferences, media choices, innovation, and other marketing phenomena [37]. A recommender system for online sales automatically suggests products and services that match the online buyer's wishes and needs based on previous buying behavior. Successful personality-based recommender systems include social matching systems (e.g., online dating systems), gift recommender systems, music recommender systems, and movie recommender systems [38]. Previous research also applied the personality to assist people in choosing jobs [37].

Past studies suggest the growing demand for technologies for recruitment in human resource management. The erecruitment system is frequently employed by companies to select candidates. Online job career portals for general purposes (such as Monster.com and Jobstreet.com) provide e-recruiting functions; these portals use the criteria of skills, experience, preference, education, salary, or their combination. An e-recruitment platform matches candidates to jobs based on a combination of keywords that define requirements [26]. Research by [24] identifies issues in erecruitment applications, which include the selection of candidates who were incompatible with the search criteria. The study by [30] showed that personality should be considered to match the right people to the appropriate job tasks.

Apart from using the e-recruitment system, social media emerged as a new platform for job searching and recruitment due to its frequent usage. Social media has become a fast and inexpensive means for employers to perform background screening of job candidates [45]. According to the 2015 Jobvite UK Social Recruitment Survey, about $92 \%$ of employers in the USA are planning or using social networks for recruitment, Linkedin being the most popular for recruitment purposes (87\%), followed by Facebook (55\%), and Twitter (47\%) [42]. By screening the social media profile of candidates, employers may gain additional information not found during the interview, including the personality [45]. Employers use personality tests to evaluate a candidate's personality. Conversely, job seekers tend to use social networking sites for their job search. Facebook is used by $67 \%$ of job seekers, $45 \%$ utilize Twitter, and $40 \%$ use Linkedin [42]. However, job candidates aim to project ideal characteristics in their answers to match their targeted job. Personality results may be misleading and may not truly reflect the personality of said individuals. Therefore, a mechanism that can instantly calculate personality through social media data is a quicker means of evaluating personality than traditional personality tests. Given the higher usage of Facebook in Malaysia than Linkedin, the present study uses Facebook as the source platform for publicly available information.

Different job positions may require different personalities. In this study, software engineering jobs are chosen as the output for Facebook personality results generated by the system. The Big Five Model and software engineering jobs were mapped by understanding the personality, skills, and job requirements for different categories of software engineering jobs [31]. Table 1 shows the different categories of software engineering jobs and their corresponding levels of personality traits in the Big Five Model. Six software engineering jobs are included, namely, management engineers, requirement engineers, system engineers, programmer, tester and implementer, and evaluator. 
Table 1: Relationship Between Software Engineering Jobs and the Big Five Model

\begin{tabular}{|c|c|c|c|c|c|c|c|}
\hline $\begin{array}{l}\text { Big Five personality } \\
\text { traits }\end{array}$ & $\begin{array}{l}\text { Personality } \\
\text { Score } \\
\text { Range }\end{array}$ & $\begin{array}{c}\text { Management } \\
\text { Engineers }\end{array}$ & $\begin{array}{l}\text { Requirement } \\
\text { Engineers }\end{array}$ & $\begin{array}{c}\text { System } \\
\text { Engineers }\end{array}$ & $\begin{array}{c}\text { Program } \\
\text { mer }\end{array}$ & $\begin{array}{c}\text { Tester } \\
\text { and } \\
\text { Impleme } \\
\text { nter }\end{array}$ & $\begin{array}{c}\text { Evalua } \\
\text { tor }\end{array}$ \\
\hline $\begin{array}{l}\text { Openness to } \\
\text { Experience }\end{array}$ & $\begin{array}{l}\text { Low } \\
\text { Medium } \\
\text { High }\end{array}$ & $\begin{array}{l}- \\
\sqrt{ } \\
-\end{array}$ & $\begin{array}{l} \\
- \\
-\end{array}$ & $\begin{array}{l}\sqrt{ } \\
- \\
-\end{array}$ & $\begin{array}{l}\sqrt{ } \\
- \\
-\end{array}$ & $\begin{array}{l}- \\
- \\
\sqrt{ }\end{array}$ & $\begin{array}{l}- \\
\sqrt{ } \\
-\end{array}$ \\
\hline Conscientiousness & $\begin{array}{l}\text { Low } \\
\text { Medium } \\
\text { High }\end{array}$ & $\begin{array}{l}- \\
\sqrt{ } \\
-\end{array}$ & $\begin{array}{l}- \\
- \\
\sqrt{ }\end{array}$ & $\begin{array}{l}- \\
- \\
\sqrt{ }\end{array}$ & $\begin{array}{l}- \\
\sqrt{ } \\
-\end{array}$ & $\begin{array}{l}- \\
\sqrt{ } \\
-\end{array}$ & $\begin{array}{l}- \\
- \\
\sqrt{ }\end{array}$ \\
\hline Extraversion & $\begin{array}{l}\text { Low } \\
\text { Medium } \\
\text { High }\end{array}$ & $\begin{array}{l}\sqrt{ } \\
- \\
-\end{array}$ & $\begin{array}{l}- \\
\sqrt{ } \\
-\end{array}$ & $\begin{array}{l}\sqrt{ } \\
- \\
-\end{array}$ & $\begin{array}{l}\sqrt{ } \\
- \\
-\end{array}$ & $\begin{array}{l}- \\
\sqrt{ } \\
-\end{array}$ & $\begin{array}{l}\sqrt{ } \\
- \\
-\end{array}$ \\
\hline Agreeableness & $\begin{array}{l}\text { Low } \\
\text { Medium } \\
\text { High }\end{array}$ & $\begin{array}{l}- \\
- \\
\sqrt{ }\end{array}$ & $\begin{array}{l}- \\
- \\
\sqrt{ }\end{array}$ & $\begin{array}{l}- \\
- \\
\sqrt{ }\end{array}$ & $\begin{array}{l}- \\
- \\
\sqrt{ }\end{array}$ & $\begin{array}{l}- \\
- \\
\sqrt{ }\end{array}$ & $\begin{array}{l}- \\
- \\
\sqrt{ }\end{array}$ \\
\hline Neuroticism & $\begin{array}{l}\text { Low } \\
\text { Medium } \\
\text { High }\end{array}$ & $\begin{array}{l}\sqrt{ } \\
- \\
-\end{array}$ & $\begin{array}{l}\sqrt{ } \\
- \\
-\end{array}$ & $\begin{array}{l}\sqrt{ } \\
- \\
-\end{array}$ & $\begin{array}{l}\sqrt{ } \\
- \\
-\end{array}$ & $\begin{array}{l}\sqrt{ } \\
- \\
-\end{array}$ & $\begin{array}{l}\sqrt{ } \\
- \\
-\end{array}$ \\
\hline
\end{tabular}

Source: [31]

Personality traits in the Big Five Model can be divided into three ranges based on [31]; these ranges are low, medium, and high. Table 1 shows that people who scored high in agreeableness and scored low in neuroticism are suitable for all of the six software engineering jobs. People who are high in openness are most appropriate as testers and implementers, whereas those who have corresponding low ratings are suitable for work as requirement engineers, system engineers, and programmers. [31] shows the relationship between jobs and personality and did not cater for identifying personality traits with Facebook features. Thus, we mapped Facebook features that contribute to personality traits and calculated the personality score for each trait.

\subsection{METHODOLOGY}

The methodology of this research is briefly discussed in this section. Fig. 1 illustrates the conceptual framework of job recommendation using FPS.

The conceptual framework of job recommendation using FPS describes the overall process involved to retrieve Facebook data and obtain the respondent's FPS. A system redirects respondents to the Facebook login page, which require the permission for apps designed to collect data from respondent's account. After login, the system extracts data in the numeric form. Respondent's data were extracted through the Facebook PHP Software Development Kit (SDK). Data is retrieved from Facebook by using tools, such as Facebook Software Development Kit, Facebook API, Graph API explorer and Facebook Query Language. SDK is one of the sources for accessing Facebook serverside API calls. In this study, Facebook PHP SDK was used for data extraction. Data such as the number of albums, wall posts, likes, friends, and others will be retrieved and stored in the database. These data will then be mapped to 
the Big Five Model. The personality of the respondent is then assessed. A computational method has been proposed to determine FPS by evaluating the collected statistics of Facebook features from the account. The respondent's FPSs for each dimension of the Big Five Model (openness, conscientiousness, extraversion, agreeableness, and neuroticism) will be calculated and displayed in percentage form. Subsequently, the FPS will be applied in the job matching process. Table 1 indicates the relationship between the Big Five Model and Software Engineering jobs, which are applied to match the most suitable jobs for the respondent's personality. Aside from assessment using online Facebook personality, respondents were also involved in offline personality assessment through the BFI Test. The BFI personality score (BFIPS) will be computed based on scores obtained in the personality test. The FPS will be compared with BFIPS and the personality result of the BFI test shall be benchmarked against the Facebook personality.

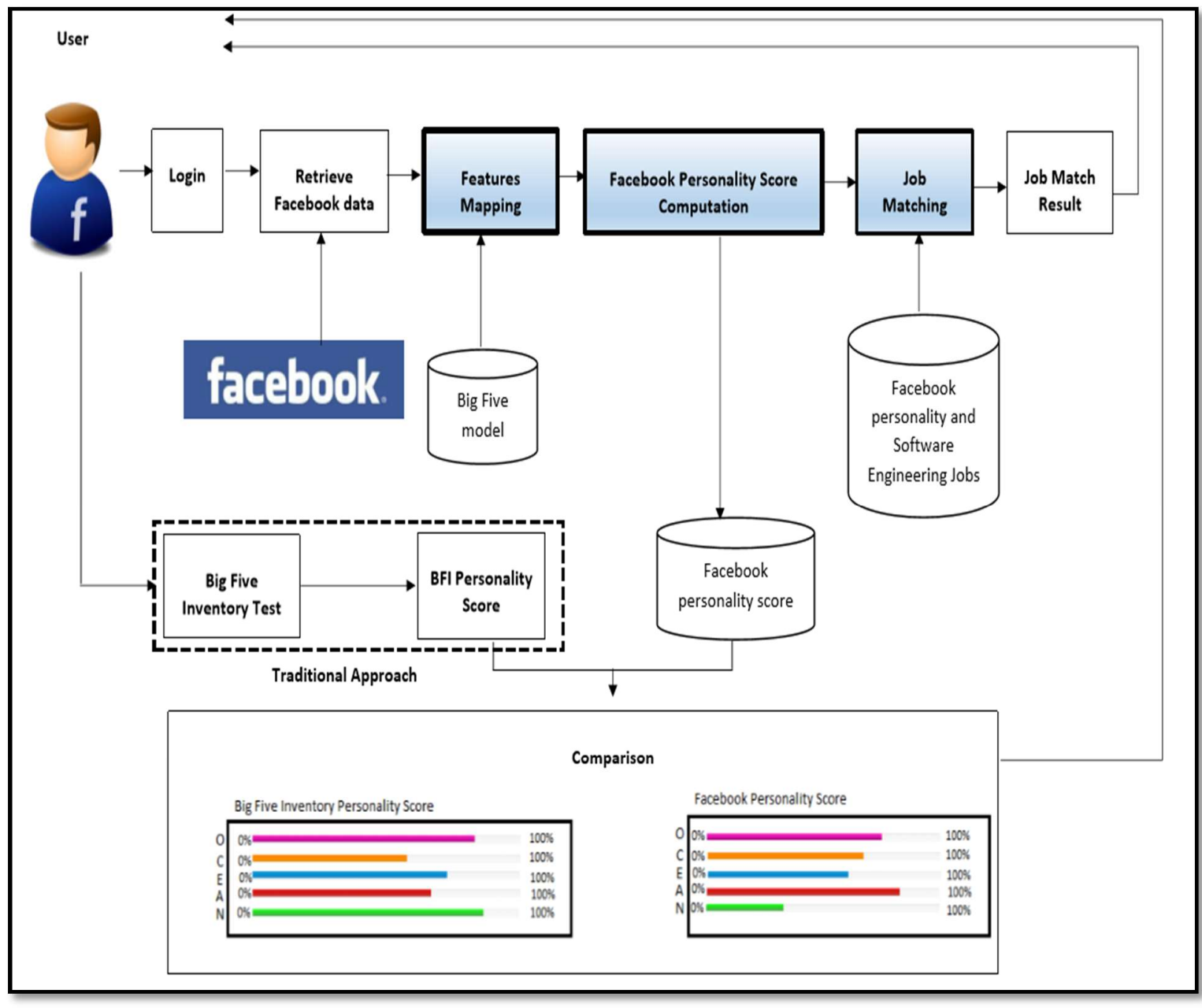

Fig. 1: Conceptual framework of job recommendation using Facebook personality scores

In the present study, we used purposive sampling technique for data collection. Fifty respondents $(56 \%$ females and $44 \%$ males) participated in the study. Respondents are selected from the Software Engineering undergraduate and postgraduate students from University Malaya, Malaysia. The students were between 20 to 30 years of age. All respondents own a Facebook account, which they use in their daily lives. The same group of respondents for the Facebook experiments was also given a set of BFI questionnaires to evaluate their personalities.

Processes highlighted in Fig. 1 shall be explained in detail. Feature mapping, FPS computation, job matching, and the BFI Test and their comparison will be covered in the following subsections. 


\subsection{Computation of FPS and Job Matching}

\subsubsection{Feature Mapping}

Feature mapping begins by examining the associations of the five dimensions of personality traits and a range of Facebook features, which include the number of albums, wall posts, likes, friends, groups, comments, and photos. The relationship between each Facebook features and personality traits in the Big Five Model is shown in Table 2.

Table 2: Relationship between each Facebook feature and the Big Five Model personality traits

\begin{tabular}{|l|l|l|l|l|l|}
\hline Facebook Feature & O & C & E & A & N \\
\hline Number of Albums & & & $+[6]$ & $+[6]$ & $+[6]$ \\
Number of Wall posts & $+[6]$ & & & & \\
Number of Likes & $+[7]$ & $-[7]$ & & $-[7][8]$ & $+[7]$ \\
Number of Friends & & & $+[1][2][4][5][6][7]$ & $+[6]$ & $-[4][6]$ \\
Number of Groups & $+[7]$ & $-[6][7]$ & $+[2][7]$ & & \\
Number of Comments & & & $+[6]$ & $+[6]$ & \\
Number of Photos in album & $+[6]$ & $+[7]$ & $+[2][6][14]$ & & \\
\hline
\end{tabular}

Note: O: Openness to experience, C: Conscientiousness, E: Extraversion, A: Agreeableness, N: Neuroticism

$(+)$ : Positive correlation and (-): Negative correlation

A positive ( + ) sign indicates the positive correlation between Facebook features and the personality traits, whereas a negative (-) sign indicates the corresponding negative correlation. In Table 2 , the numbers inside the brackets [X] represent previous studies which discovered the existence of the relationship between Facebook features and personality traits.

Table 2 shows that the number of albums has a positive relationship with extraversion, agreeableness, and neuroticism. A high number of albums suggests that the person has a high degree of extraversion, agreeableness, and neuroticism. The number of wall posts is positively correlated with openness to experience. The number of likes is positively correlated with the personality traits of openness to experience and neuroticism, but a negative correlation is shown for conscientiousness and agreeableness. People with high levels of extraversion and agreeableness are expected to have a high number of friends. However, those who scored high in neuroticism have a negative relationship with the number of friends. This result indicates that a high level of neuroticism shows few friends in their Facebook profile. Moreover, the number of groups has a positive relationship with openness to experience and extraversion and a negative correlation with conscientiousness. Table 2 also suggests that an increase in the number of comments corresponds to increase in extraversion and agreeableness.

\subsubsection{Computation of FPS}

FPS computation follows feature mapping. A proposed scoring mechanism calculates Facebook personality. A few steps are implemented for such calculation (see Fig. 2). 


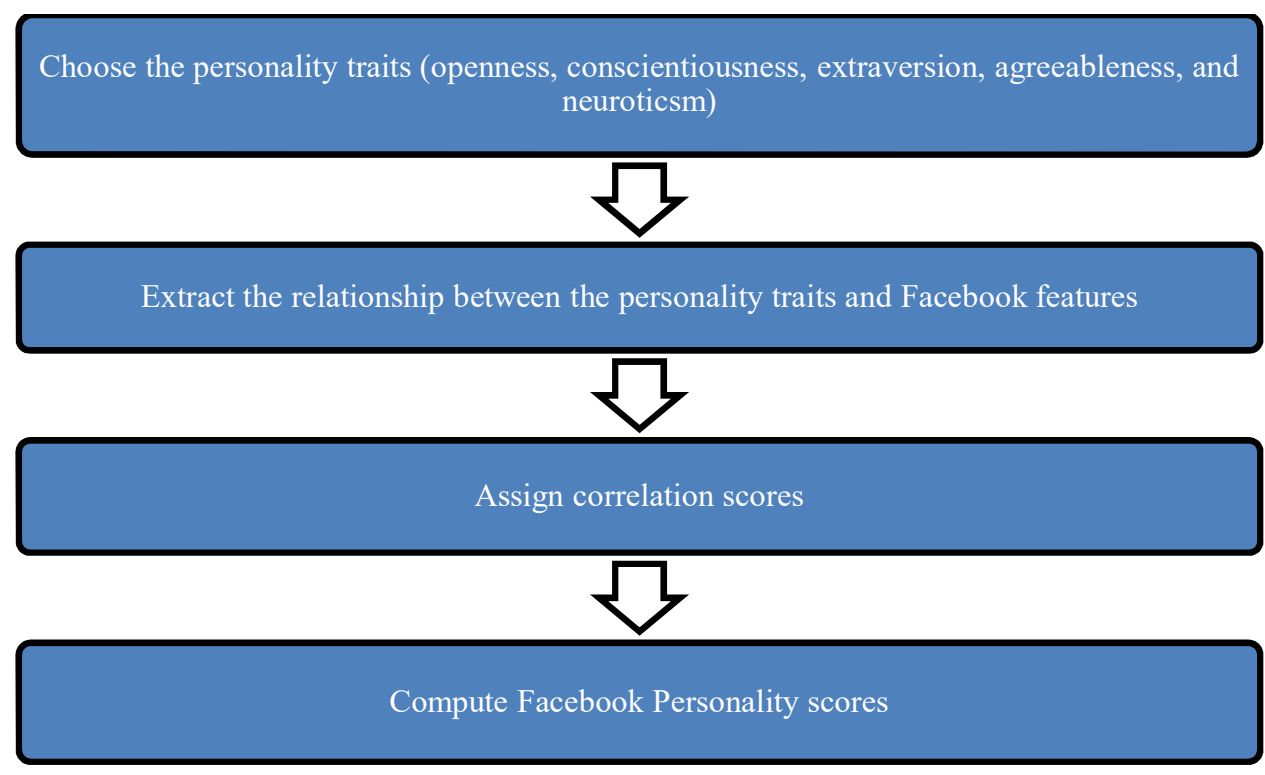

Fig. 2: Steps for Facebook Personality Score Computation

Fig. 2 shows that FPS computation begins by choosing personality traits from the Big Five Model. The five personality traits are openness, conscientiousness, extraversion, agreeableness, and neuroticism. The relationship between personality traits and Facebook features are shown in Table 2. A positive or negative relationship exists between Facebook features and personality. After retrieving Facebook data, a numeric range is set ranging from low to high for Facebook features data (as shown in Table 3). Correlation scores were then assigned using a Likert scale from 1 to 3, and FPS is computed.

The computed FPS is displayed in the form of percentage to indicate the strength of the personality traits of the respondent under low, medium, or high levels from the Big Five Model. High FPS indicates strong personality trait. FPS calculation is performed for all five personality traits in the Big Five Model.

The system retrieves Facebook features data in the numeric form. Thus, data do not need to be assigned to low, medium, or high category. The range (low, medium and high) corresponds to the characteristics of the Big Five Model [16]. To categorize the features, three ranges have been set and the Facebook feature range is determined using Formula (1):

$$
\text { Facebook feature range }=\frac{\text { Highest number of each feature-Lowes number of each feature }}{3}
$$

The sum of each Facebook features is collected from all respondents and averaged based on Formula (1). The range of numbers for each Facebook feature is set and summarized as shown in Table 3. 
Table 3: Facebook feature range for each software engineering student

\begin{tabular}{|l|l|l|l|}
\hline Facebook Features & Low & Medium & High \\
\hline Number of Likes & $0-301$ & $302-602$ & $>602$ \\
Number of Friends & $0-527$ & $528-1055$ & $>1055$ \\
Number of Wall Posts & $0-559$ & $510-1117$ & $>1117$ \\
Number of Albums & $0-40$ & $41-79$ & $>79$ \\
Number of Photos in album & $0-3442$ & $344-6885$ & $>6885$ \\
Number of Comments & $0-134$ & $135-268$ & $>268$ \\
Number of Groups & $0-23$ & $24-46$ & $>46$ \\
\hline
\end{tabular}

Table 3 shows the Facebook feature ranges for software engineering students based on low, medium, and high usage. After determining the Facebook range for all the features, a range can be used to categorize the features extracted in numeric format.

In the present study, correlation scores are assigned using a Likert scale that ranges from 1 to 3 . The Likert scale is used to measure three levels (1: Low, 2: Medium, and 3: High). A feature in the low range will be computed as 1, 2 for the medium range, and 3 for the high range. For example, if the respondent has 1100 friends on Facebook, the range of the features Friends will be considered high with a score of 3 . However, scores can be counted in a reverse direction. For reversed features (with - sign) as shown in Table 2, the score is distributed in a reverse direction. For instance, people with high conscientiousness will have fewer likes. If the respondent only has 200 likes on Facebook, the range falls under the low range but the score is assigned as 3 .

FPS is calculated after assigning correlation scores. FPS for each trait is computed according to the proposed Formula (2) below.

$$
\text { Facebook Personality Score for X trait }(F P S)=\sum_{i=1}^{n} \frac{\left(x_{1}+x_{2}+x_{3}+\cdots+x_{i}\right)}{3 n} X 100 \%
$$

Note:

- $n$ represents the upper bound of summation and refers to number of Facebook features exist relation with the particular personality traits

- $i$ represents the index of summation

- $x_{i}$ represents an indexed variable of each successive term in series and refers to the scores given for each feature

Table 4 shows an example of how a personality trait is computed based on the extracted Facebook data. We have selected the agreeableness trait from the Big Five Model, where the relationships between agreeableness and Facebook features are extracted as shown in Column 2, and column 4 shows the correlation scores according to the range of each feature.

Table 4: Sample personality traits for agreeableness of a respondent

\begin{tabular}{|l|c|c|c|}
\hline Facebook Features & $\begin{array}{c}\text { Relationship between } \\
\text { Agreeableness and } \\
\text { Facebook Features }\end{array}$ & Range & $\begin{array}{c}\text { Correlation } \\
\text { Scores }\end{array}$ \\
\hline Number of Albums & Positive(+) & High & 3 \\
Number of Wall Posts & Not related & - & - \\
Number of Likes & Negative(-) & Mow & 3 \\
Number of Groups & Positive(+) & - & 2 \\
Number of Comments & Not related & Medium & - \\
Number of Photos in Album & Positive(+) & - & 2 \\
\hline
\end{tabular}




$$
\text { Facebook Personality Score for Trait }=\sum_{i}^{3} \frac{(3+3+2+2)}{3(4)} X 100 \%=83 \%
$$

From Table 4, four Facebook features (albums, likes, friends, and comments) are found to be correlated with the personality traits of Agreeableness. For positive features, scores given are in the forward direction (positive), whereas negative features are assigned scores in the reverse direction (negative). From the example above, the respondent exhibits $83 \%$ agreeableness through their Facebook data. This score indicates that the respondent is a highly agreeable person. In this research, results below $30 \%$ are considered as belonging to the low level, while those above $70 \%$ are considered as high. The remaining features are considered as medium level.

\subsubsection{Model}

A model is created to map Facebook personality and software engineering jobs. The model shows the link among Facebook features, the Big Five Model, and software engineering jobs.

This study contributes to the development of a model of Facebook-based personality identification and software engineering job recommendation as shown in Fig. 3. This figure describes the relationship between Facebook features and the Big Five Model based personality traits. The study has successfully mapped FPSs to the specified software engineering jobs through the Big Five Model.

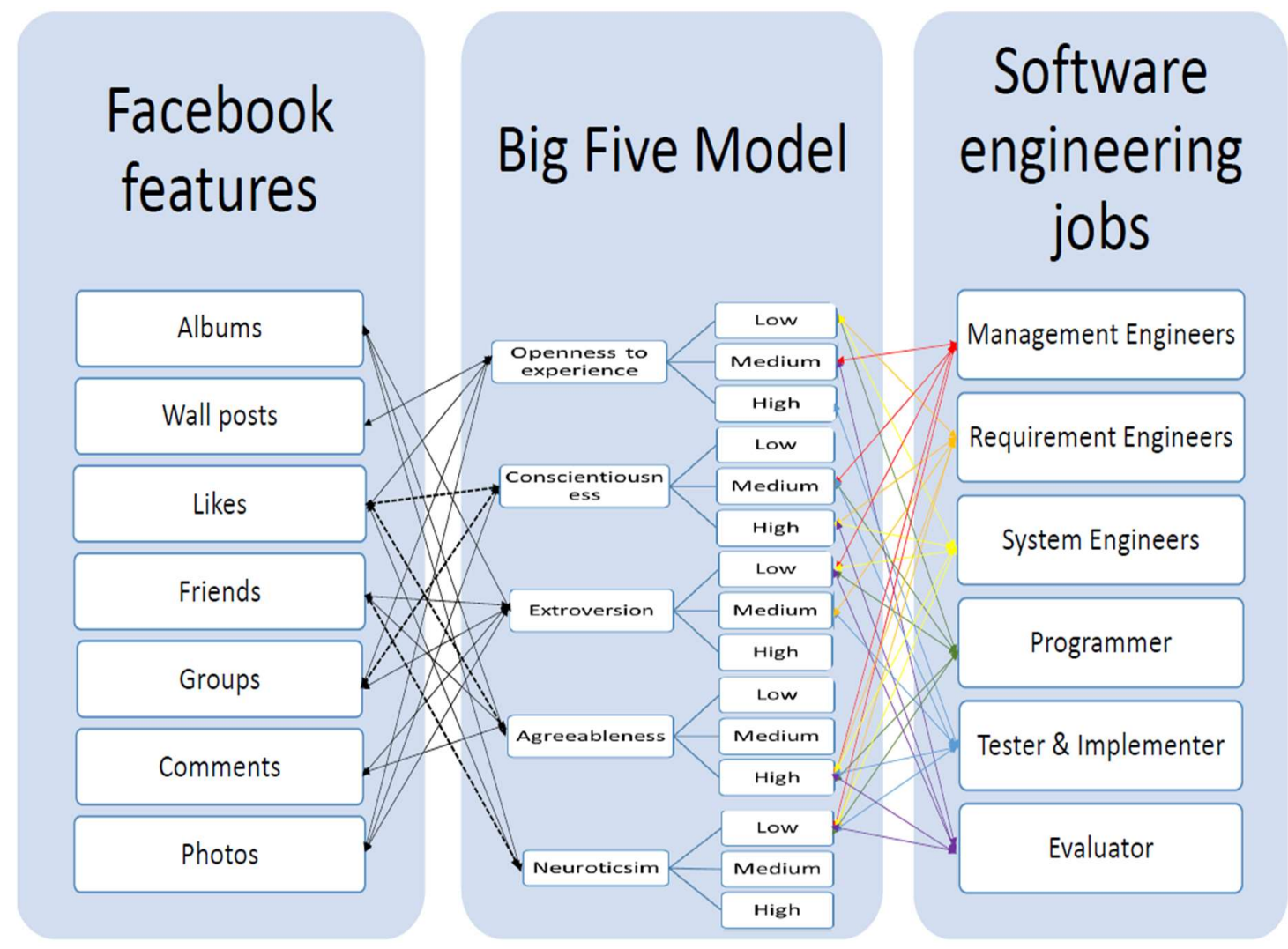

Fig. 3: Model that maps Facebook personality and software engineering jobs

The model linked three important elements in this study, namely, the Facebook features, the Big Five Model, and the software engineering jobs. Facebook features include the seven frequently used features, whereas the Big Five Model consists of five personality traits, which include openness to experience, conscientiousness, extraversion, agreeableness, and neuroticism. We then mapped an individual's personality to their Facebook features. Each feature is correlated with the personality traits, either positively or negatively. Fig. 3 shows that a solid line links Facebook features and personality traits, which indicates a positive relationship. By contrast, a dashed line indicates 
the contrary. Fig. 3 indicates that the group feature is positively related to openness to experience in the Big Five Model whereas Likes is negatively related to Conscientiousness. The relationship between personality traits and Facebook features has been explained fully in Section 3.1.1. Furthermore, we linked personality traits in three levels for different jobs in software engineering such as management engineers, requirement engineers, system engineers, programmers, testers and implementers, and evaluators. Each colored line represents a different job. For example, those who fall under the medium level of Openness to experience are suitable as management engineers.

\subsubsection{Job Matching}

After determining the respondent's Facebook personality, the top three careers that fit the respondent's personality will be recommended (see Fig. 4). For the example, Fig. 6 shows that a respondent displayed 58\% openness, 67\% conscientiousness, 30\% extraversion, $83 \%$ agreeableness, and 33\% neuroticism. This person will be categorized with a low level of extraversion; medium levels of openness to experience, conscientiousness, and neuroticism, and a high level of agreeableness. The relationship of the Big Five Model and software engineering jobs displayed in Table 1 is employed to match the most suitable jobs for the respondent's personality. Through the intersection of sets, jobs will be recommended based on the level of the respondent's personality for each trait. Six software engineering jobs are included, which are management engineers, system engineers, requirement engineers, programmers, testers and implementers, and evaluators. The system will map the Facebook personality result with software engineering jobs.

Fig. 4 shows that a respondent with a medium level of Openness is suitable for employment as a management engineer, and evaluator. The respondent with the medium level of conscientiousness, match with management engineer, programmer, tester, and implementer. A respondent with low extraversion is suitable for jobs like management engineer, system engineer, programmer, and evaluator. All of the six software engineering jobs are suitable to the respondent's high agreeableness. None of the software engineering jobs fit the medium level neuroticism. The three most suitable jobs recommend to the respondent are based on the frequency of jobs that match the personality arranged. Jobs are arranged from most frequent to least frequent. Jobs with the same frequency shall be sorted further. In this example, management engineer occurred four times, thus, ranking in the first place. Programmer is ranked second and evaluator ranked third. Fig. 4 shows the discussed sample of a job match result.

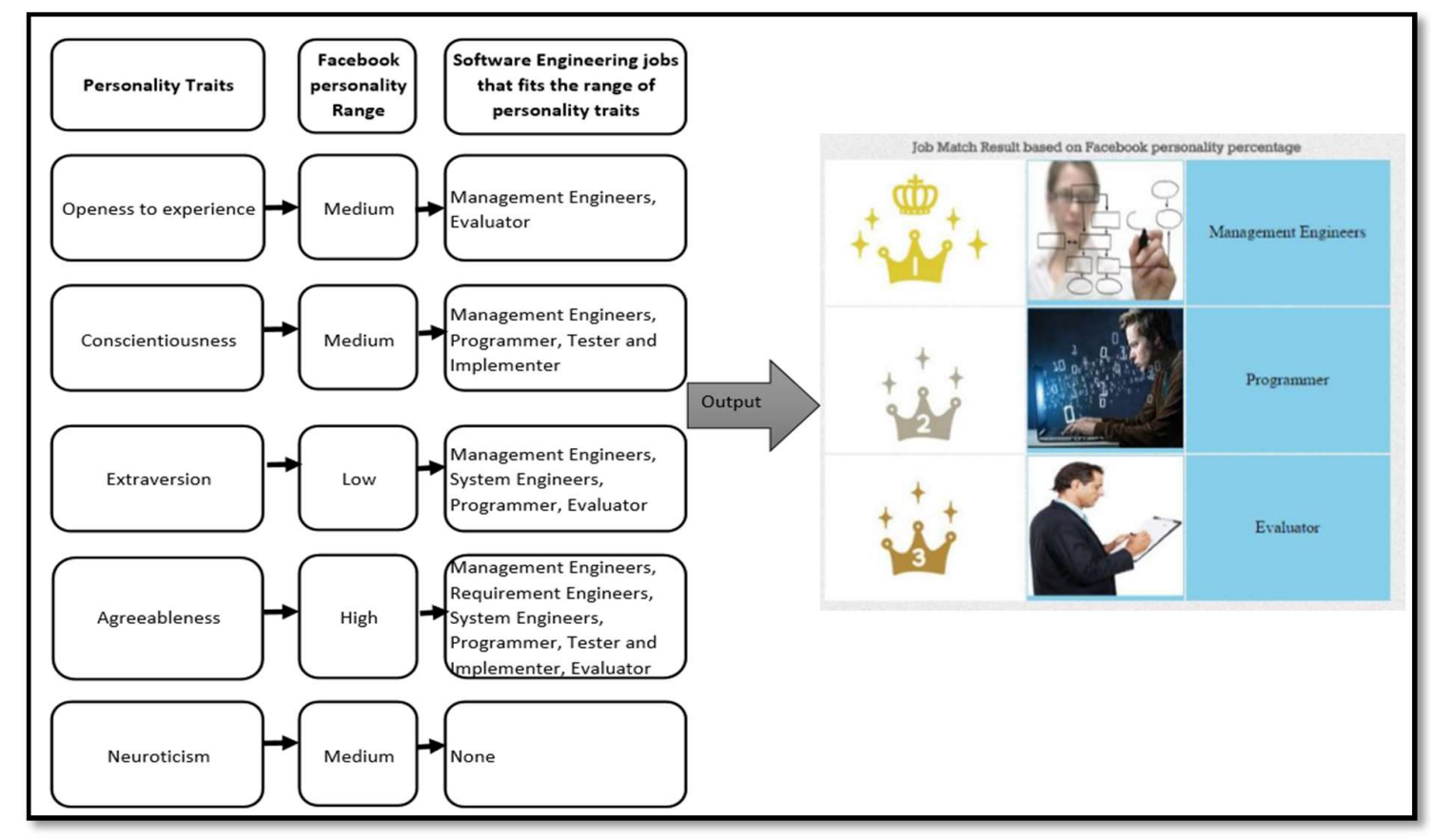

Fig. 4: Sample of job match result

Note: Job ranking is sorted in ascending order from (1) Management Engineers, (2) Requirement Engineers, (3) System Engineers, (4) Programmer, (5)Tester and Implementer and (6)Evaluator. 


\subsection{Traditional Approach}

\subsubsection{Big Five Inventory Test}

In previous studies, the BFI test was used to identify an individual's personality. To evaluate the results of the BFI Test, 44 questions were categorized into five personality domains (openness, conscientiousness, extraversion, agreeableness, and neuroticism). The BFI test uses a Likert scale ranging from 1 to 5, wherein 1 represents "strongly disagree" and 5 "strongly agree." The evaluation of the BFI Test results was set by computation of scale scores as shown below. The BFI test result was computed by averaging the scale scores of items in each personality domain and displayed in the final result in percentage. This test is similar to the Facebook personality test where a respondent will be categorized based on percentage scores. To determine whether a respondent is low, medium or high level in a particular personality domain, we consider those below $30 \%$ as low level, those above $70 \%$ as high, and those between $30 \%$ to $70 \%$ as medium level [16][29].

Table 5: Computation of scale score using BFI Test

\begin{tabular}{|l|l|}
\hline Personality Traits & Questions that correspond to personality traits \\
\hline Openness & $5,10,15,20,25,30,35 \mathrm{R}, 40,41 \mathrm{R}, 44$ \\
\hline Conscientiousness & $3,8 \mathrm{R}, 13,18 \mathrm{R}, 23 \mathrm{R}, 28,33,38,43 \mathrm{R}$ \\
\hline Extraversion & $1,6 \mathrm{R}, 11,16,21 \mathrm{R}, 26,31 \mathrm{R}, 36$ \\
\hline Agreeableness & $2 \mathrm{R}, 7,12 \mathrm{R}, 17,22,27 \mathrm{R}, 32,37 \mathrm{R}, 42$ \\
\hline Neuroticism & $4,9 \mathrm{R}, 14,19,24 \mathrm{R}, 29,34 \mathrm{R}, 39$ \\
\hline
\end{tabular}

Source: [6]

Note: $\mathrm{R}$ represents reversed item, where the scale score is counted in reverse fashion

In Table 5, a number represents the number of questions; those without $\mathrm{R}$ are positive items, and those with $\mathrm{R}$ are reversed items. $\mathrm{R}$ represents the reverse-scored items where a scale score is given in the reverse fashion, that is, a score of 1 becomes 5, 2 becomes 4, 3 remains 3, 4 becomes 2, and 5 becomes 1 .

The score for each question is distributed according to the group of personality domain indicated by the question. As shown above, eight questions with the number 4, 9R, 14, 19, 24R, 29, 34R, and 39 belong to the group for Neuroticism. To compute neuroticism personality result in BFI, each score collected for the questions in the Neuroticism group will be added and divided by eight. The average score of the questions in a group of the personality domain is the personality result and the same process is applied for the four remaining personality domains.

\subsection{Comparison}

The computation of FPS (discussed at 3.1.2) and the BFIPS (discussed at 3.2.1) were compared. Both personality scores are calculated using the five personality traits, and ranging from $0 \%$ to $100 \%$. This comparison indicates the accuracy of FPS and suggests whether personality can be predicted with high accuracy through Facebook usage. Fig. 5 shows an example of the comparison of the BFIPS and FPS of an individual.

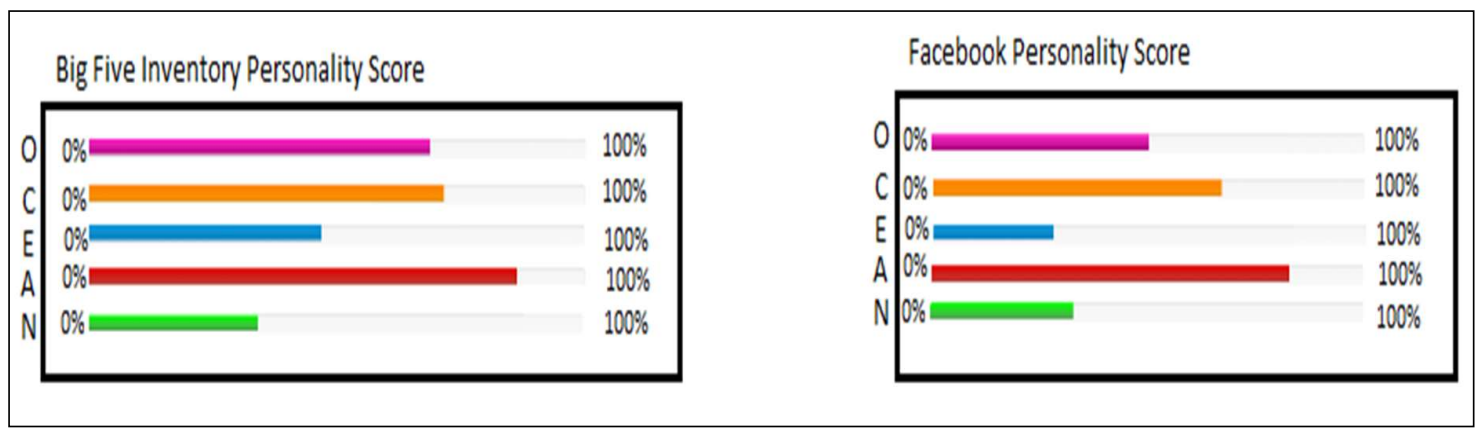

Fig. 5: Example of comparison of Big Five Inventory Personality Score and Facebook Personality Score of an individual 


\subsection{EXPERIMENTAL RESULTS AND DISCUSSIONS}

\section{1 $\quad$ FPS}

This section discusses the experimental results of the FPSs and the BFIPS of 50 respondents with software engineering background. Each of their Facebook data is extracted to determine their personalities. Fig. 6 shows the FPS of a respondent, where the respondent obtain $58 \%$ openness to experience, $67 \%$ conscientiousness, $30 \%$ extraversion, $83 \%$ agreeableness, and $33 \%$ neuroticism.

The scores imply that the respondent falls in the medium range for openness to experience, conscientiousness, and neuroticism; in the low range for extraversion; and in the high range for agreeableness. The result of the BFIPSs is similar to FPSs, where the scores range from $0 \%$ to $100 \%$ for five personality traits.

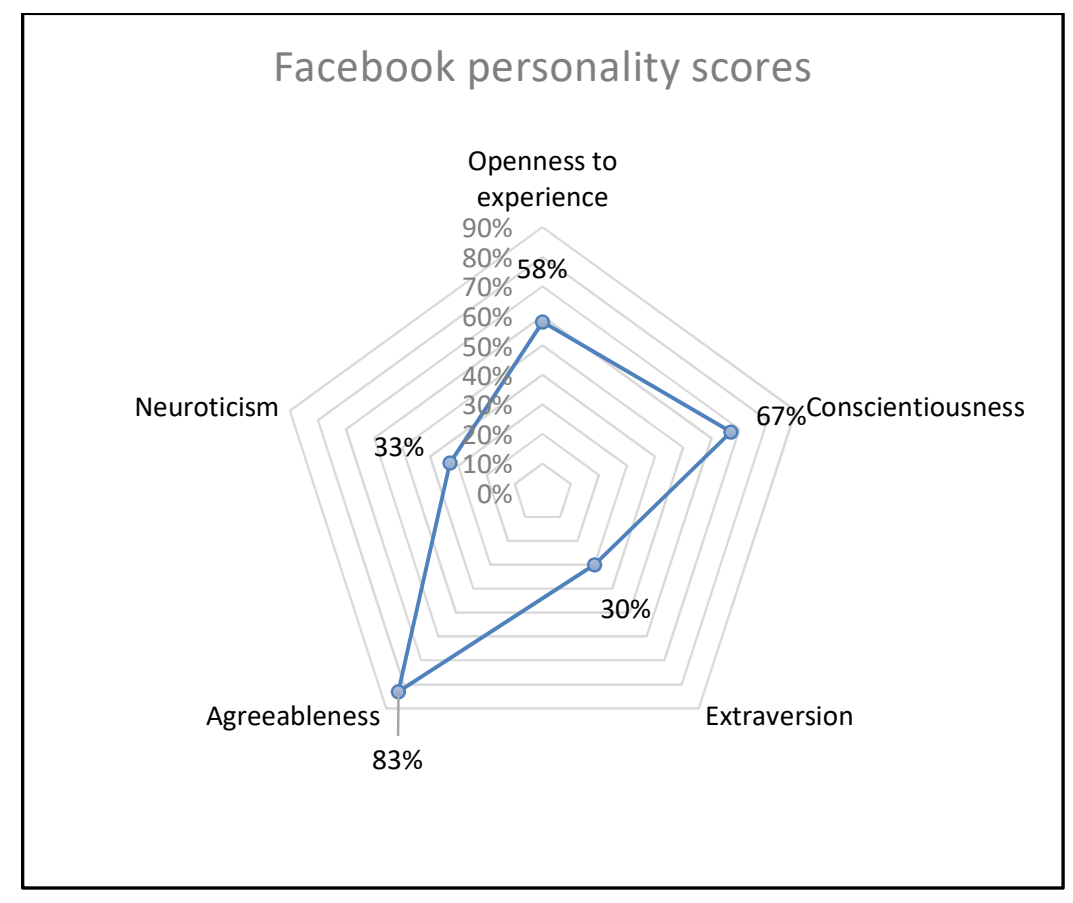

Fig. 6: Sample FPS score of an individual

Fig. 7 shows the BFIPSs of a respondent. The respondent managed to obtain $70 \%$ openness to experience, $73 \%$ conscientiousness, $50 \%$ of extraversion, $87 \%$ of agreeableness, and $42 \%$ neuroticism. BFIPS is computed similarly to FPS. 


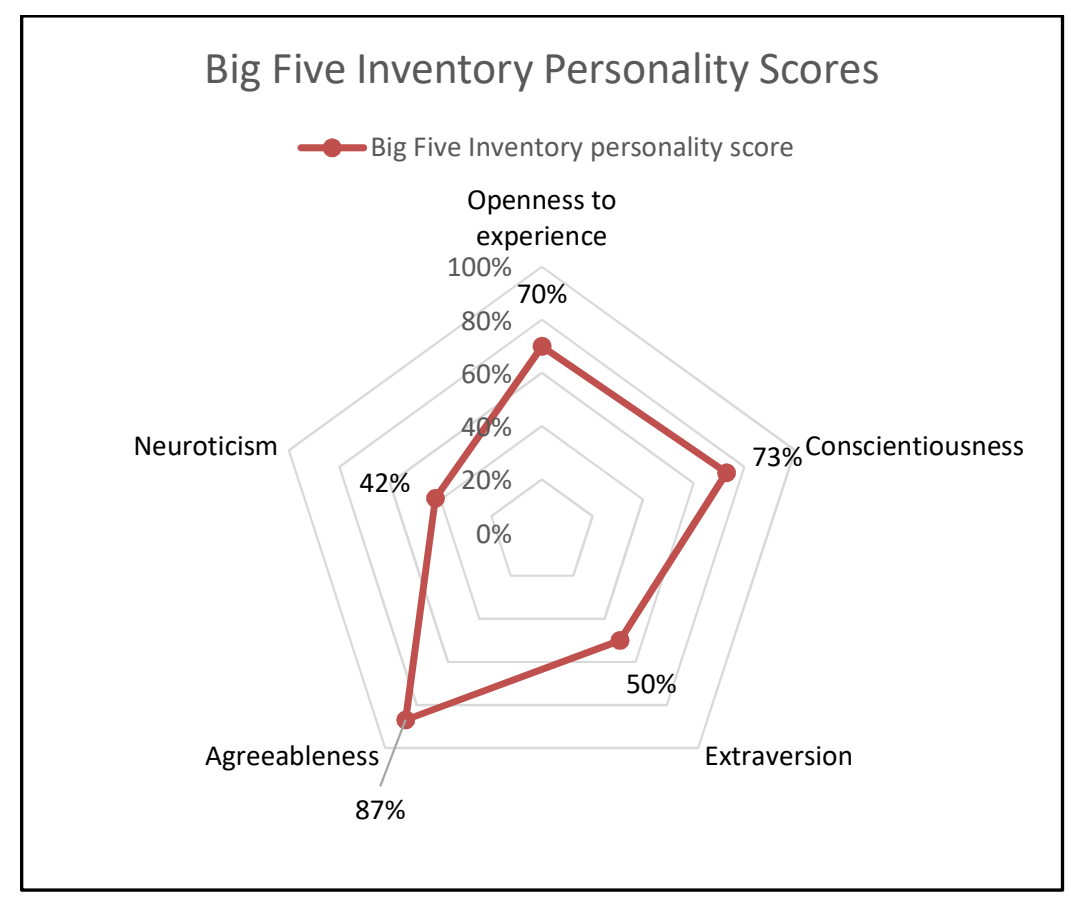

Fig. 7: Sample of Big Five Inventory personality scores of an individual

\subsection{Comparison of Scores}

This section presents a comparison of scores between Facebook and the BFI Test. Compassion was made on 30 and 50 respondents (Figs. 8 and 9, respectively) and shall be explained further in the following sections. Two different results are required, which provides an improved understanding of the changes in a respondent's pattern and behavior through graphs and through the sample size increase. Thus, we obtained a clear picture of the behavior of Facebook users to predict Facebook personality more accurately. Both results provide additional information regarding the respondent's personality trait through Facebook and the BFI Test.

\subsubsection{Thirty respondents}

Fig. 8 suggests that personality scores assessed using Facebook show a similar pattern to the BFIPSs. Personality scores for the BFI Test are higher in percentage than personality scores for Facebook except for openness. This finding indicates that people are more open in Facebook than in the BFI test. This finding is supported by [3], which revealed that people engaged in social networking tend to be more open to experience online compared to real life. Regarding the time spent on Facebook, people with high conscientiousness behave indifferently from those with high openness [7]. Other personality traits, such as extraversion, agreeableness, and neuroticism are low on Facebook because such traits were not found to influence social media usage; hence their lower scores in Facebook [12]. Based on Fig. 8, the average personality score for Facebook is $57.12 \%$ and the average BFI Test is $61.75 \%$. The FPS accuracy is calculated using the formula: (average personality score for Facebook/average Big Five Inventory Test score) $* 100 \%$. Thus, the FPS accuracy is $(57.12 / 61.75) * 100=92.5 \%$. 


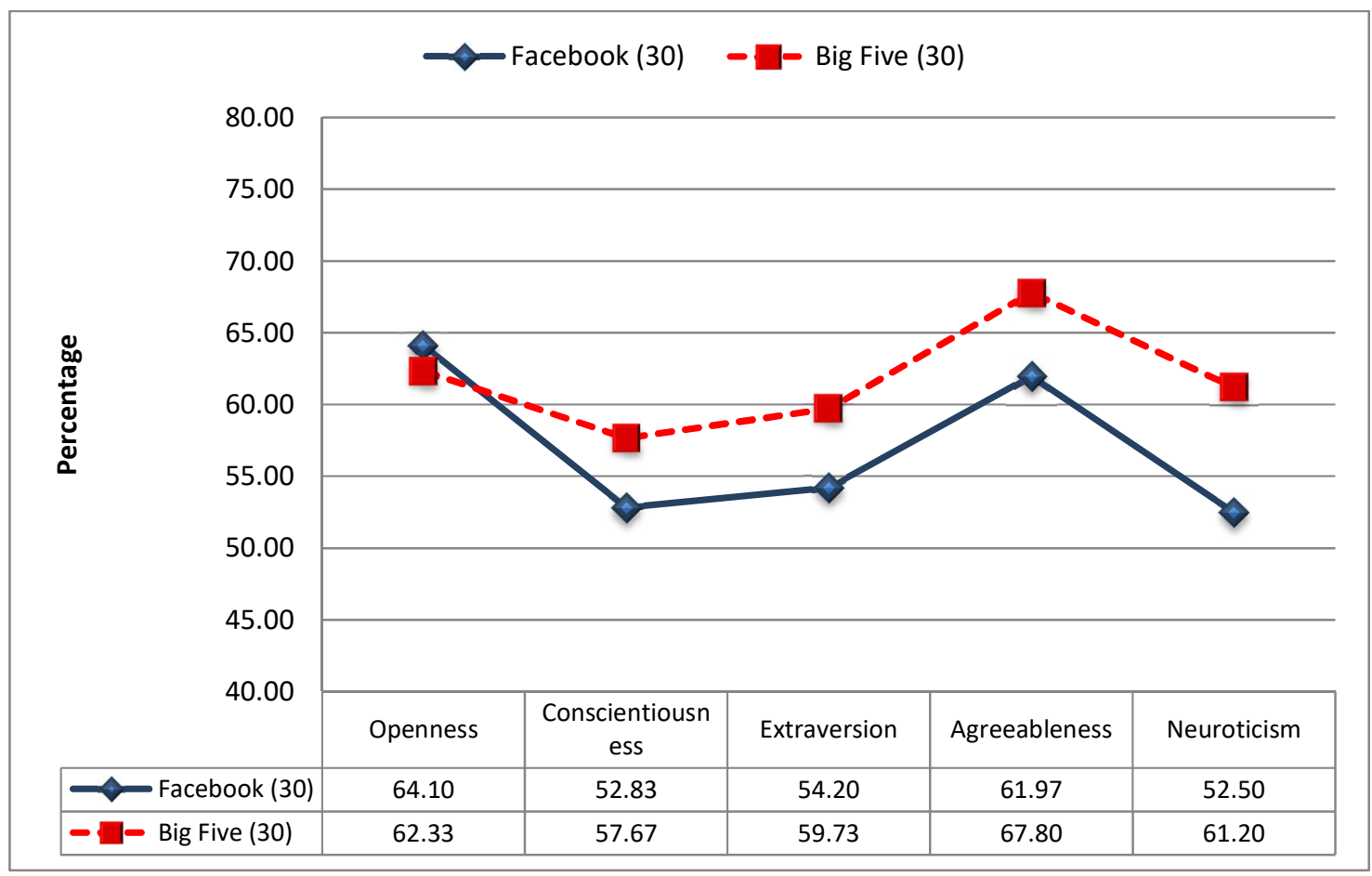

Fig. 8: Comparison of mean of FPS and BFI personality scores of 30 respondents

\subsubsection{Fifty respondents}

Fig. 9 shows the overall personality scores of 50 respondents for the FPSs and the BFIPSs. The average personality score for Facebook is $58.72 \%$ and the average for BFI Test is $63.07 \%$. Thus, the FPS accuracy is $(58.72 / 63.07) * 100$ $=93.1 \%$. The FPS accuracy slightly increased from $92.5 \%$ to $93.1 \%$ as the sample size increase from 30 to 50 respondents.

A comparison was made between the two different sample sizes of 30 and 50 respondents. Two sample sizes better illustrate the movement and changes in personality as the population increases. If the respondents behave similarly in the two sample sizes, we can predict that respondents from a larger population will display the same personality results. Generally, the FPS and BFI scores of the two sample sizes display the same pattern (see Fig. 8 and Fig. 9). As the sample size increases from 30 to 50, the movement of personality scores in the graph for 50 people is similar and consistent with the previous sample. The same situations were present in personality scores for the openness which were slightly higher in Facebook than in the BFI. With the increase in sample size, respondents were found to behave in a similar pattern as the pattern of personality scores for both the BFI and FPS remain the same. Therefore, the sample size used is justified for this study. 


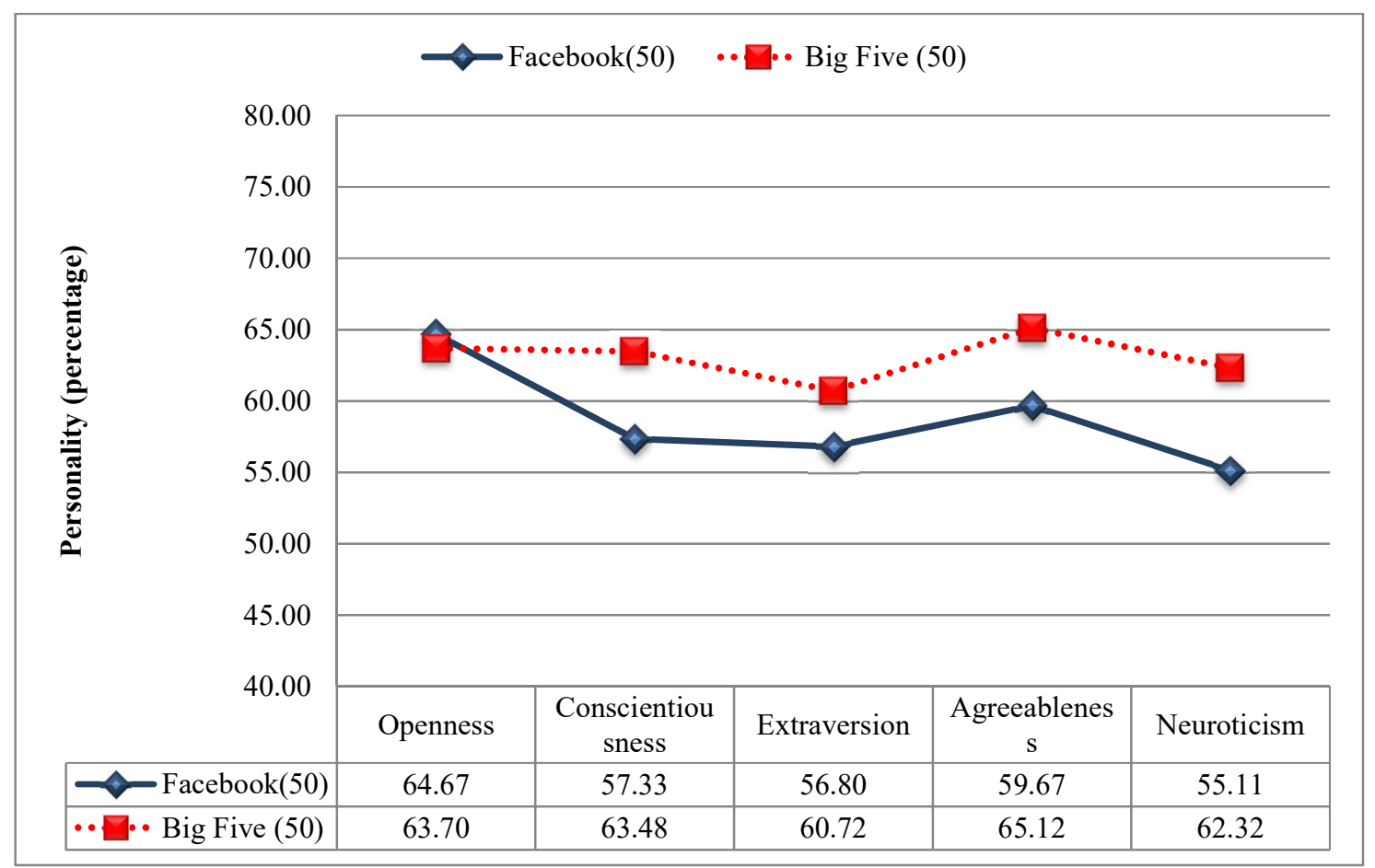

Fig. 9: Comparison of mean of FPS and BFI personality scores of 50 respondents.

\subsubsection{Cohen's Kappa}

Aside from using accuracy to determine the reliability of the Facebook personality result, Cohen's kappa was also employed in this study. This value can determine the degree of agreement of the results from the FPSs and the BFIPS obtained under similar personality measurement conditions. Interrater reliability is a measure for observing the agreement between two raters (in this study, refers to the BFI score and FPS) on the assignment of categories of a categorical variable. One of the interrater reliability values is Cohen's Kappa, which ranges from -1.00 to 1.00 . Large numbers represent better reliability and values near or less than zero indicate that agreement is due to chance alone. Calculation of Cohen's Kappa is performed using the following formula:

$$
k=(\operatorname{Pr}(a)-\operatorname{Pr}(e)) /(1-\operatorname{Pr}(e))
$$

where $\operatorname{Pr}(\mathrm{a})$ is the actual observed agreement and $\operatorname{Pr}(\mathrm{e})$ is the chance agreement [33].

Cohen's kappa determines the presence of an agreement between two personality scores. Table 6 shows the kappa values and their corresponding level of agreement. Table 7 lists the evaluation of kappa value for two scores, namely, BFIPS and FPS. 
Table 6: Kappa Value and Level of Agreement

\begin{tabular}{|l|l|}
\hline Kappa Value & Level of Agreement \\
\hline$<0$ & Less than chance agreement \\
\hline $0.01-0.20$ & Slight agreement \\
\hline $0.2-0.40$ & Fair agreement \\
\hline $0.41-0.60$ & Moderate agreement \\
\hline $0.61-0.80$ & Substantial agreement \\
\hline $0.81-0.99$ & Almost perfect agreement \\
\hline Reference: $[34]$ & \\
\hline
\end{tabular}

Table 7: Kappa value using BFI personality score and Facebook personality score

\begin{tabular}{llcc}
\hline Personality domain & & & Value \\
\hline Openness & N of Valid Cases & & 50 \\
Conscientiousness & Measure of Agreement & Kappa & .567 \\
Extraversion & Measure of Agreement & Kappa & .691 \\
Agreeableness & Measure of Agreement & Kappa & .751 \\
Neuroticism & Measure of Agreement & Kappa & .579 \\
& Measure of Agreement & Kappa & .453 \\
\hline
\end{tabular}

Table 7 suggests that the mean kappa value obtained by averaging the kappa value of five personality domains is 0.608. According to [34], the level of agreement of kappa analyzed with a value less than 0 suggests a value lower than chance agreement, wherein 0.01 to 0.20 is considered slight, 0.2 to 0.4 is fair, 0.41 to 0.60 is moderate, 0.6 to 0.80 is substantial, and over 0.81 indicates almost perfect agreement. All the personality domains in Table 7 had moderate to the substantial agreement, with kappa values that range from 0.453 to 0.751 . Table 7 also indicates that extraversion obtained a value of 0.751 falls under the substantial agreement. Results showed a substantial agreement between extraversion and two personality metrics. The respondents somewhat agree that both personality scores were measured similarly and both personality score results achieved were identical under similar assessment conditions. This result is consistent with [47], wherein accuracy was strongest for extraversion and lowest for neuroticism. Previous research showed that neuroticism is difficult to detect in all zero-acquaintance contexts [47]. In conclusion, the kappa value has substantial agreement with the mean value of 0.608 and the personality result in this study is reliable.

\subsection{BIF and FPS Metrics}

In this study, a personality score has been created to compute Facebook personality. This computation method differs with the traditional way of predicting personality. Traditionally, the BFI test is used to evaluate an individual's personality result whereas this research uses Facebook data. Table 8 below compares both methods. 
Table 8: Comparison between BIF Test method and Facebook method

\begin{tabular}{|c|c|}
\hline Big Five Inventory & Facebook \\
\hline \multicolumn{2}{|c|}{ Differences } \\
\hline Requests user to answer a survey of 44 questions & $\begin{array}{l}\text { Request user to use the implemented system to } \\
\text { retrieve publicly available Facebook features }\end{array}$ \\
\hline $\begin{array}{l}\text { A score scales from } 1 \text { to } 5 \text { (Strongly disagree to } \\
\text { strongly agree) }\end{array}$ & A score scales from 1 to 3 ( low, medium, high) \\
\hline Accuracy depends on user honesty & $\begin{array}{l}\text { Accuracy depends on user's activeness in } \\
\text { Facebook }\end{array}$ \\
\hline \multicolumn{2}{|c|}{ Similarities } \\
\hline Use Big Five Personality Model & Use Big Five Personality Model \\
\hline
\end{tabular}

The computation of the BFI Test shows the personality results (in percentage) for five personality traits of the Big Five Model. However, the accuracy of personality results of the BFI Test is affected by the user's caution while giving their responses.

Conversely, the Facebook method differs from the BFI method. In this research, users are required to connect with their Facebook account to retrieve publicly accessible information using Facebook API. Data on Facebook features were retrieved as numbers were evaluated and given a score. Scores range from 1 to 3 and are distributed as low to high.

The scoring mechanism of Facebook is used to calculate the FPS through the retrieved Facebook data. Finally, the Facebook personality result is displayed in percentage form. Both methods use the Big Five Model in gauging a user's personality.

\subsection{Benchmarking of Personality Score}

In this research, personality evaluated through Facebook and the personality result are both related to software engineering jobs. This research extended the [31] study of software engineering jobs and personality. The research of [31] used the Big Five Model and Cognitive Ability to predict personality with software engineering jobs but omits the use of any social media data in evaluating personality. Other studies also used social media in identifying personality through social media profiles. Previous work predicted Facebook personality through analysis of behaviors on Facebook [6]. However, [31] did not utilize the information in calculating FPS.

Few similarities and differences exist between the FPS and the study by Sodiya (2007). Table 9 shows the comparisons made between this current research and [31].

Table 9: Comparison of Facebook Personality Score and Sodiya (2007)

\begin{tabular}{|c|c|c|}
\hline & $\begin{array}{l}\text { Facebook Personality } \\
\text { Score }\end{array}$ & Sodiya(2007) \\
\hline Maps personality and jobs & $\sqrt{ }$ & $\sqrt{ }$ \\
\hline Recommends job for software engineering & $\sqrt{ }$ & $\sqrt{ }$ \\
\hline Uses the Big Five Model as personality model & $\sqrt{ }$ & $\sqrt{ }$ \\
\hline Computes Personality Score from Facebook & $\sqrt{ }$ & $\mathrm{X}$ \\
\hline Utilizes social media data to predict personality & $\sqrt{ }$ & $\mathrm{X}$ \\
\hline $\begin{array}{l}\text { Compares the Big Five Inventory and Facebook personality } \\
\text { score }\end{array}$ & $\sqrt{ }$ & $\mathrm{X}$ \\
\hline
\end{tabular}


The present study and Sodiya (2007) managed to map jobs in software engineering based on personality and recommend jobs related to the respondent's own personality. Several differences are noted between both studies. Sodiya used the Big Five Model and Cognitive Ability, whereas only the Big Five Model was used in this study. Moreover, the present study uses social media (Facebook), whereas Sodiya did not involve any social media data. Another obvious difference is that this study mapped jobs using FPS, whereas Sodiya's study mapped jobs using questionnaire scores. With regard to significant contribution, this research utilized social media data to predict personality. A comparison has also been made for both the BFIPS and the FPSs.

\subsection{CONCLUSION AND FUTURE WORKS}

This study retrieved required information and identified personality from user-generated Facebook data. This research managed to create awareness among users regarding their own personality. Collected Facebook data are mapped accordingly and personality scores for each trait were computed. Another significant contribution of this study is the proposed scoring mechanism for calculating the Facebook personality scores of an individual. In addition, a model has been created to map the Facebook features with the BIF Test and software engineering jobs.

Employers may utilize the Facebook personality result to recruit the most suitable candidates for job positions. Facebook reduces the time spent on interview sessions and on observing the suitability of candidates. Assigning people to the right position in an organization improves job efficiency and enhances job satisfaction.

Furthermore, this research can be useful for marketing purposes, where the features such as Likes or Groups can facilitate the identification of individual preferences and personality. By using such information, salespeople can easily introduce and recommend their products to targeted customers. Personality prediction through online social media usage can also be applied to those who encounter difficulties in choosing the courses for their enrolment in higher education institutions.

Additionally, understanding an individual's personality can be applied in crime prevention. For example, the Neuroticism in the Big Five Personality Model can measure a person's emotional instability. People with high levels of neuroticism display the characteristics of feeling anxious, insecure, and depressed. Therefore, those who scored higher on neuroticism may pose a higher risk of committing crimes. This information will be extremely beneficial for a psychologist and for the respondent to gain awareness of their own strengths and weaknesses.

Certain limitations are present in this work. First, the accuracy of the Facebook personality result depends heavily on the activeness of the user on Facebook. Second, the accuracy of the BIF Test result is affected by the candor of respondents. Another constraint is the limited number of software engineering jobs used in the analysis. This study can be expanded beyond software engineering jobs. Future studies should also consider other Facebook features in gauging personality.

\section{ACKNOWLEDGEMENTS}

We would like to take this opportunity to thank University of Malaya research grant (RP028D-14AET) for funding this research.

\section{REFERENCES}

[1] E. Dawson, "Facebook Profiles and Usage as Indicators of Personality", 2011.

[2] Y. Amichai-Hamburger and G. Vinitzky, "Social network use and personality", Computers in Human Behavior, Vol. 26 No. 6, 2010, pp. 1289-1295.

[3] C. Ross, E. S. Orr, M. Sisic, J. M. Arseneault, M. G. Simmering and R. R. Orr, "Personality and motivations associated with facebook use", Computers in Human Behavior, Vol. 25 No. 2, 2009, pp. 578 586. 
[4] D. Quercia, R. Lambiotte, D. Stillwell, M. Kosinski and J. Crowcroft, "The personality of popular facebook users", in Proceedings of the ACM 2012 conference on computer supported cooperative work, ACM, February 2012, pp. 955-964.

[5] J. Golbeck, C. Robles, and K. Turner, "Predicting personality with social media ", in PCHI'11 Extended Abstracts on Human Factors in Computing Systems, ACM, May 2011, pp. 253-262.

[6] C. Sumner, A. Byers and M. Shearing, "Determining personality traits \& privacy concerns from facebook activity", Black Hat Briefings, Vol. 11, 2011, pp. 197-221.

[7] Y. Bachrach, M. Kosinski, T. Graepel, P. Kohli and D.Stillwell, "Personality and patterns of Facebook usage", in Proceedings of the 3rd annual ACM web science conference, ACM, June 2012, pp. 24-32.

[8] A. Eftekhar, C. Fullwood and N. Morris, "Capturing personality from Facebook photos and photo-related activities: How much exposure do you need?", Computers in Human Behavior, Vol. 37, 2014, pp. 162-170.

[9] G. Farnadi, S. Zoghbi, M. F. Moens and M. De Cock, "Recognising personality traits using facebook status updates", in Proceedings of the workshop on computational personality recognition (WCPR13) at the $7^{\text {th }}$ international AAAI conference on weblogs and social media (ICWSM13), June 2013.

[10] D. J. Hughes, M. Rowe, M. Batey and A. Lee, "A tale of two sites: Twitter vs. Facebook and the personality predictors of social media usage", Computers in Human Behavior, Vol. 28 No. 2, 2012, pp. 561-569.

[11] N. Mahadi, "13.3 million M'siansare Facebook users", BorneoPostOnline.com, from http://www.theborneopost.com/2013/06/16/13-3-million-msians-are-facebook-users/, 16 June 2013.

[12] N. Ozguven and B. Mucan, "The relationship between personality traits and social media use", Social Behavior and Personality: an international journal, Vol. 41 No. 3, 2013, pp. 517-528.

[13] S. Bai, B. Hao, A. Li, S. Yuam, R. Gao and T. Zhu, "Predicting Big Five personality traits of microblog users", in Web Intelligence (WI) and Intelligent Agent Technologies (IAT), 2013 IEEE/WIC/ACM International Joint Conferences, Vol. 1, November 2013, pp. 501-508.

[14] T. Kuo and H. L. Tang, "Relationships among personality traits, Facebook usages, and leisure activities-A case of Taiwanese college students", Computers in Human Behavior, Vol. 31, 2014, pp.13-19.

[15] A. P. Chioqueta and T. C. Stiles, "Personality traits and the development of depression, hopelessness, and suicide ideation", Personality and Individual Differences, Vol. 38 No. 6, 2015, pp. 1283-1291.

[16] D. P. Count and T. D. S. H. Skill, "Does Personality Count?", 2012.

[17] K. Suresh, "Social Media participatory cultures - A study of the dynamics between user personality and facebook use", International Journal of Current Research, Vol. 5 Issue 4, 2013, pp. 925-930.

[18] W. Merrin, Media Studies 2.0., Routledge, 2014.

[19] Facebook, "Facebook Products", Facebook.com, from http://newsroom.fb.com/products, 2014.

[20] Facebook, "Facebook newsroom", Facebook.com, from http://newsroom.fb.com/company-info, 2016.

[21] StatisticBrain, "Facebook statistics", Facebook.com, from http://www.statisticbrain.com/facebookstatistics, 27 January 2015.

[22] F. Sarsar and S. Harmon, "Facebook as an online learning environment: perceptions of undergraduate students", in Society for Information Technology \& Teacher Education International Conference, Vol. 1, March 2011, pp. 715-720. 
[23] Institute of Labour Market Information \& Analysis, "Top 5 jobs", ILMIA, from http://www.ilmia.gov.my/welcome, 2015.

[24] A. Singh, C. Rose, K. Viswewariah, V. Chenthamarakshan and N. Kambhatla, "Prospect: A System for Screening Candidates for Recruitment", in Proceeding of $19^{\text {th }}$ ACM International Conference on Information and Knowledge Management (CIKM'10), ACM, 2010, pp. 659-668.

[25] L. F. Capretz and F. Ahmed, "Making sense of software development and personality types", IT professional, Vol. 12 No. 1, 2010, pp. 6-13.

[26] S. T. Al-Otaibi and M. Ykhlef, "A survey of job recommender systems", International Journal of the Physical Sciences, Vol. 7 No. 29, 2012, pp. 5127-5142.

[27] M. B. Donellan, F. L. Oswald, B.M. Baird and R. E. Lucas, "The mini-IPIP scales: tiny-yet-effective measures of the Big Five factors of personality", Psychology assessment, Vol. 18 No. 2, 2006, pp.192.

[28] M. K. Mount, M. R. Barrick, S. M. Scullen, and J. Rounds, "Higher-order dimensions of the big five personality traits and the big six vocational interest types", Personnel Psychology, Vol. 58 No. 2, 2005, pp. 447-478.

[29] P. J. Howard and J. M. Howard, " The Big Five Quickstart: An Introduction to the Five-Factor Model of Personality for Human Resource Professionals", 1995.

[30] J. Malinowski, T. Keim, O. Wendt and T. Weitzel, "Matching People and Jobs: A Bilateral Recommendation Approach", in Proceeding of the $39^{\text {th }}$ Annual Hawaii International Conference on System Sciene, IEEE, 2006, pp. 137-145.

[31] A. S. Sodiya, H. O. D. Longe, S. A. Onashoga, O. Awodele and L. O. Omotosho, "An Improved Assessment of Personality Traits in Software Engineering", Interdisciplinary Journal of Information, Knowledge \& Management, Vol. 2, 2007.

[32] A. R. A. Rashid, S. Hussin and A. T. Putih, "Career Development and Unemployment Problems in Malaysia: Crisis of Education and Training ", Utusan Publications, 2005, pp. 14.

[33] M. L. McHugh, "Interrater realibility:the kappa statistics", Biochemica medica, Vol. 22 No. 3, 2012, pp. 276-282.

[34] A. J. Viera and J.M. Garrett, "Understanding interobserver agreement: the kappa statistic", Fam Med, Vol. 37 No. 5, 2005, pp. 360- 363.

[35] J. B. D'Souza and S. Saelee, "The Influence of Personality Traits in Relation to Choice of Major in University", in Proceedings of World Business and Economic Research Conference 24-25 February, 2014.

[36] National Education Statistics: Higher Education Sector 2013, "Number and percentage of Malaysian Graduates Employment Status According to Type of HEIS (2013-2013)”, Kementerian Pendidikan Tinggi , from http://www.mohe.gov.my/web_statistik/Perangkaan\%20Pendidikan\%20Tinggi\%20Negara\%202013.pdf

[37] R. Hu, "Design and user issues in personality-based recommender system", in Proceedings of the fourth ACM conference on Recommender systems, 2010, pp. 357-360.

[38] M.A.S. Nunes and R.Hu, "Personality-based recommender system: an overview", in Proceedings of the sixth ACM conference on Recommender system, 2012, pp. 5-6.

[39] P. Nieken and S. Stomer, "Personality as predictor of occupational choice: Empirical evidence from Germany", in Proceedings of World Business and Economic Research Conference 24-25 February, 2010. 
[40] D.H. Kluemper, P.A. Rosen and K.W. Mossholder, "Social networking websites, personality ratings, and the organizational context: More than meets the eye?", in Journal of Applied Social Psychology, Vol. 42 No. 5, 2012, pp. 1143- 1172 .

[41] K. Kumar, "Linking the 'Big Five' Personality Domains to Organizational Citizenship Behaviour”, in International Journal of Psychological Studies, Vol. 1 No. 2, 2009, pp. 73-81.

[42] JobVite, “Job Seeker Nation Study”, JobVite.com, from http://www.jobvite.com/wpcontent/uploads/2015/01/jobvite_jobseeker_nation_2015.pdf, 14 February 2015.

[43] Z.Hanapi and M. S. Nordin, “Unemployment among Malaysia Graduates: Graduates' attributes, lecturer's competency and quality of education", Procedia-Social and Behavioral Science, Vol. 112, 2014, pp. 10561063.

[44] JobStreet, "Employers Rank Soft Skills above Academics”, JobStreet.com, from http://www.jobstreet.com.my/career-resources/employers-rank-soft-skills-academics/\#.Uw4CZyhbRkM, 26 Februarry 2014.

[45] A.Broughton, B.Foley, S.Ledermaier and A. Cox, "The use of social media in the recruitment process", Acas, from $h t t p: / / w w w . a c a s . o r g . u k / m e d i a / p d f / 0 / b /$ The-use-of-social-media-in-the-recruitment-process.pdf

[46] E. Faliangka, A.Tsakalidis and G.Tzimas, "An integrated e-recruitment system for automated personality mining and applicant ranking”, Internet research, Vol. 22 No. 50, 2012, pp. 551-568.

[47] M.D.Back, J.M.Stopfer, S.Vazire, S.Gaddis, S.C.Schmukle, B.Egloff and S.D.Gosling, "Facebook profiles reflect actual personality, not self-idealization", Psychological Science, Vol. 21 No. 3, 2010, pp. 371-374. 